\title{
Early Basal Ganglia Hyperperfusion on CT Perfusion in Acute Ischemic Stroke: A Marker of Irreversible Damage?
}

\author{
V. Shahi, J.E. Fugate, D.F. Kallmes, and A.A. Rabinstein
}

\begin{abstract}
BACKGROUND AND PURPOSE: CT perfusion scans are often used in acute stroke evaluations. We aimed to assess the outcome of areas of basal ganglia hyperperfusion on CTP in patients with acute ischemic stroke.

MATERIALS AND METHODS: We retrospectively reviewed the medical records and brain imaging of 139 patients presenting with acute stroke who underwent CTP for consideration of endovascular recanalization. Hyperperfusion was assessed qualitatively and defined as a matched region of increased cerebral blood flow and cerebral blood volume. CTA was used to locate arterial occlusion. Follow-up imaging was used to determine whether regions of hyperperfusion at baseline became infarcted or developed hemorrhage. Angiographic imaging was assessed to determine the presence or absence of early venous opacification.
\end{abstract}

RESULTS: Six patients (4.3\%) demonstrated hyperperfusion in the basal ganglia of the affected side ( 4 in the lenticular nucleus and 2 in the caudate). In all cases, the area of hyperperfusion ultimately proved to be infarcted. All patients had received intravenous thrombolysis before the CTP. CTA at the time of CTP showed middle or distal M1 occlusion but patency of the proximal M1 and A1 segments. Intracranial hemorrhage was noted in 2 of these 6 patients at follow-up.

CONCLUSIONS: Acute basal ganglia hyperperfusion in patients with stroke may indicate nonviable parenchyma and risk of hemorrhagic conversion.

ABBREVIATION: $\mathrm{PH}=$ parenchymal hemorrhage

$C^{\top}$ perfusion scans are widely applied to assess ischemia (low flow) and allow the identification of potentially salvageable brain tissue (ischemic penumbra) with delineation of irreversibly damaged brain (core infarct). ${ }^{1,2}$ While definitions vary considerably, most studies using CTP define nonviable regions of brain tissue by the reduction in cerebral blood flow $^{3}$ or reduced cerebral blood volume. ${ }^{4,5}$ Regions of hyperperfusion (increased CBF and increased CBV) before recanalization are generally not considered areas that are infarcted or at risk of infarction. ${ }^{6,7}$

Nonviable brain typically has disturbed autoregulation. Following recanalization, a phenomenon called "luxury" perfusion-elevated CBF in areas of infarcted tissue beyond what is needed for metabolic needs_can occur. ${ }^{8,9}$ These areas may be at risk for hemorrhage, ${ }^{8,9}$ but this has not been a consistent finding. ${ }^{10}$ In our practice, we observed cases in which areas of basal

Received December 6, 2013; accepted after revision February 13, 2014.

From the Mayo Medical School (V.S.) and Departments of Neurology (J.E.F., A.A.R.) and Radiology (D.F.K.), Mayo Clinic, Rochester, Minnesota.

Please address correspondence to Jennifer E. Fugate, DO, Department of Neurology, Mayo Clinic, 200 First St SW, Rochester, MN 55905; e-mail:

fugate.jennifer@mayo.edu

http://dx.doi.org/10.3174/ajnr.A3935 ganglia hyperperfusion on CTP obtained during acute stroke evaluation evolved to completed infarction on subsequent imaging. We hypothesized that baseline CTP can demonstrate areas of hyperperfused but nonviable parenchyma, if local recanalization, either spontaneous or in response to lytic therapy, occurs before CTP imaging. The purpose of this study was to determine the prevalence of regions of hyperperfusion on baseline CTP and their rate of progression to infarction and reperfusion hemorrhage at follow-up.

\section{MATERIALS AND METHODS}

This study was approved by the Mayo Clinic Institutional Review Board. We performed a retrospective review of consecutive patients presenting with acute ischemic stroke who underwent CTP for consideration of endovascular treatment from September 2008 to October 2012. In general, we apply CTP to select patients for endovascular therapy either after intravenous thrombolysis without improvement (CTP is performed toward the conclusion of the infusion) or when patients are ineligible for intravenous thrombolysis. A neuroradiologist (D.F.K., with 24 years of experience) reviewed all CTP images to identify patients with areas of hyperperfusion. Identified cases were evaluated and confirmed by 
Baseline patient characteristics

\begin{tabular}{|c|c|c|c|c|c|c|c|c|c|c|c|}
\hline & Age/Sex & HTN & CAD & DM & A. fib & Initial BP, (mm Hg) & NIHSS & MCA Territory & Time to CTP (mins) ${ }^{a}$ & TICI Grade & $\mathrm{ICH}$ \\
\hline 1 & $79 / F$ & $\mathrm{Y}$ & $\mathrm{N}$ & $\mathrm{N}$ & $\mathrm{N}$ & $125 / 63$ & 16 & $R$ & 343 & $2 a$ & $\mathrm{Y}$ \\
\hline 2 & $61 / M$ & $\mathrm{~N}$ & Y & $\mathrm{N}$ & $Y$ & $116 / 70$ & 21 & L & 187 & $2 b$ & Y \\
\hline 3 & $61 / \mathrm{M}$ & $\mathrm{N}$ & $\mathrm{N}$ & $\mathrm{N}$ & $\mathrm{N}$ & $114 / 72$ & 17 & $\mathrm{R}$ & 125 & $2 b$ & $\mathrm{~N}$ \\
\hline 4 & $66 / M$ & Y & $\mathrm{N}$ & $\mathrm{N}$ & Y & $155 / 99$ & 21 & L & 190 & $2 b$ & $\mathrm{~N}$ \\
\hline 5 & $61 / F$ & Y & $N$ & $\mathrm{~N}$ & $\mathrm{~N}$ & $140 / 80$ & 20 & $\mathrm{R}$ & 230 & 0 & $\mathrm{~N}$ \\
\hline 6 & $65 / F$ & Y & $\mathrm{N}$ & $\mathrm{N}$ & $\mathrm{N}$ & $146 / 92$ & 16 & L & 210 & $N A^{b}$ & $\mathrm{~N}$ \\
\hline
\end{tabular}

Note:- HTN indicates hypertension; CAD, coronary artery disease; DM, diabetes mellitus; A. fib, atrial fibrillation; BP, blood pressure; ICH, intracranial hemorrhage; R, right; L, left; $Y$, yes; N, no; NA, not applicable.

${ }^{a} \mathrm{TICl} 0$ indicates no perfusion; $2 \mathrm{a}$, perfusion of $\leq 50 \%$ of MCA distribution; $2 \mathrm{~b}$, perfusion of $>50 \%$ of MCA distribution; 3 , full perfusion.

${ }^{\mathrm{b}}$ Endovascular therapy was not attempted because of large core on CTP.
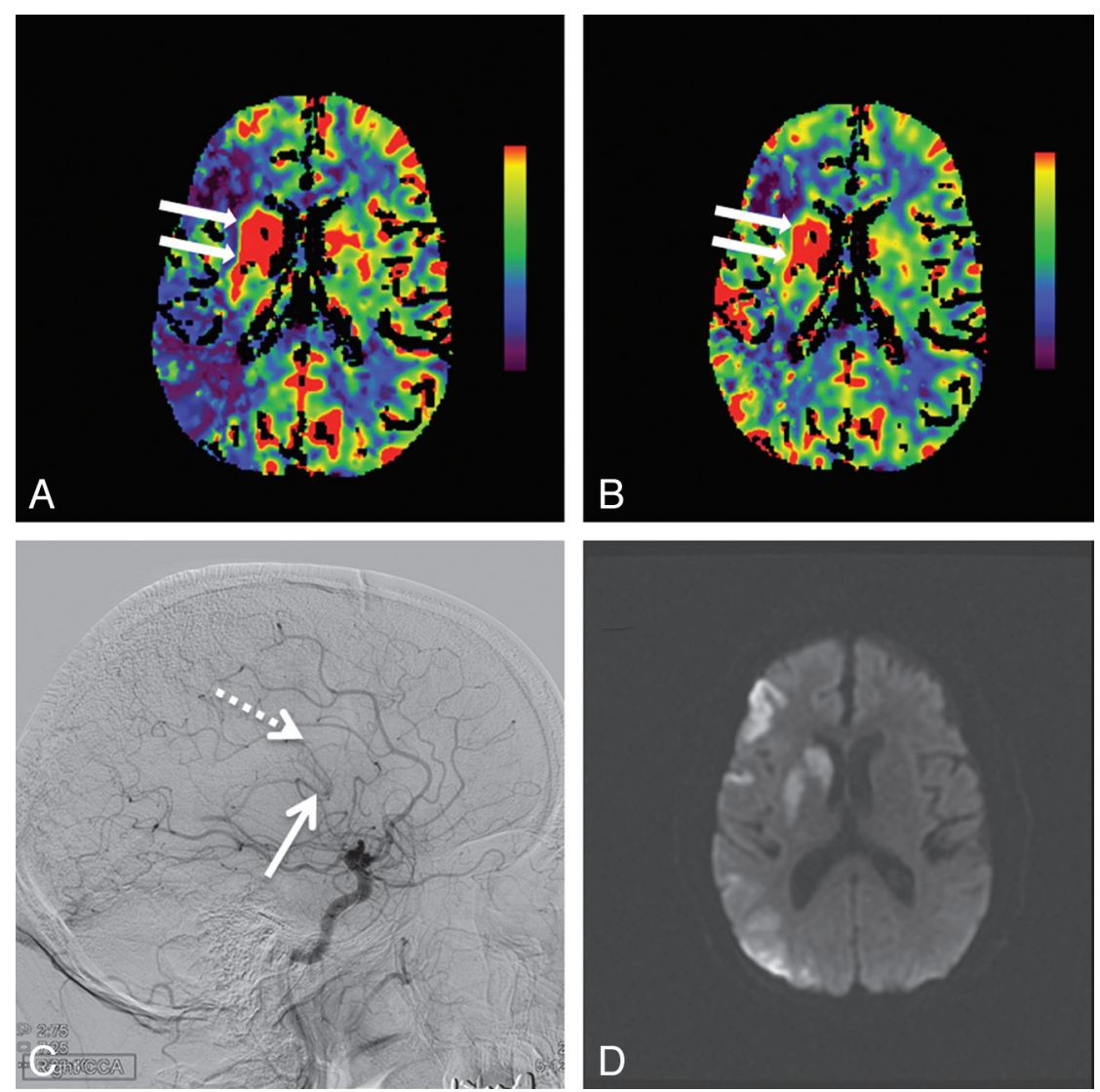

FIG 1. Hyperperfusion in the right basal ganglia is demonstrated by increased cerebral blood flow (arrows, $A$ ) and increased cerebral blood volume (arrows, $B$ ). Also seen is a large region of right middle cerebral artery territory ischemia as evidenced by reduced $C B F(A)$ with relative preservation of CBV $(B)$. Lateral digital subtraction angiogram from a right carotid artery injection demonstrates early opacification of the basal vein of Rosenthal (solid arrow, $C$ ) and the internal cerebral vein (dashed arrow, C). Follow-up MR imaging 12 hours later shows restricted diffusion indicating infarction of the right basal ganglia, along with patchy cortical infarctions in the right MCA territory $(D)$.

a vascular neurologist (A.A.R., with 12 years of experience). Perfusion parametric maps were created by using a deconvolution technique. Fifty milliliters of contrast dye was injected at $7 \mathrm{~mL} / \mathrm{s}$, followed by $50 \mathrm{~mL}$ normal saline through an 18-ga right antecubital IV catheter. Scanning took place over 71 seconds total with 20 scans every 1.5 seconds followed by 6 scans every 7.5 seconds. Our postprocessing was done using a single value deconvolution algorithm from GE software licensed to Siemens (Erlangen, Germany). This allowed the creation of time-to-peak, mean transit time, cerebral blood flow, and cerebral blood volume maps. For the purpose of this study, hyperperfusion was defined as an increase in both $\mathrm{CBF}$ and $\mathrm{CBV}$ in the same brain region compared with the contralateral region. The site of vascular occlusion was determined by using CTA images obtained at the time of CTP imaging. Conventional angiography was assessed to determine early venous opacification.

Final area of infarction was determined by using follow-up brain imaging and was compared with the initial CTP to evaluate the outcome of the region of hyperperfusion. At our institution, every patient who undergoes attempted IV or intraarterial stroke revascularization therapy has follow-up brain imaging at or within 24 hours, usually with noncontrast head CT. Further MR imaging is performed at the discretion of the treating clinicians. No patients were excluded on the basis of lack of follow-up brain imaging. Hemorrhagic events were classified according to European Cooperative Acute Stroke Study II definitions. ${ }^{11}$ Hemorrhagic infarction consists of petechial hemorrhage without mass effect, parenchymal hemorrhage $(\mathrm{PH})$ 1 indicates blood clot occupying $\leq 30 \%$ of the infarcted area with slight mass effect, and $\mathrm{PH} 2$ is blood clot in $>30 \%$ of the infarcted area with substantial space-occupying effect. ${ }^{11}$ Thrombolysis in Cerebral Infarction grades were used to evaluate the degree of reperfusion. ${ }^{12}$

\section{RESULTS}

One hundred thirty-nine patients were reviewed. Endovascular stroke therapy was attempted in approximately half of patients $(n=67,48 \%)$. Six patients $(4.3 \%)$ had CTP evidence of hyperperfusion of the basal ganglia on the side of arterial occlusion (Table). Among these 6 patients, median age at diagnosis was 63 years (range, 61-79). The median NIHSS score was 18.5 (range, 16-21). All patients received intravenous rtPA before CTP. Three of the patients had effacement of the gray-white differentiation of the basal ganglia on noncontrast head CT. Median time to intravenous thrombolysis was 87.5 minutes (range, 56135 minutes). Median time from stroke-symptom onset to CTP was 200 minutes (range, 125-343 minutes).

The median diameter of the region of hyperperfusion was 2.73 $\mathrm{cm}$ (range, 1.67-3.89 cm). On CT angiography, all patients had 
arterial occlusions of the minor distal aspect of the M1 segment of the middle cerebral artery, distal to at least a portion of the medial lenticulostriate arteries. Four occlusions involved the midportion of the M1, and 2 patients had occlusions at the junction of the middle and distal one-third of the M1 segment. The Al segment was patent in all cases. Five patients also underwent digital subtraction angiography showing early venous opacification in 2 cases, one in the deep venous system and one
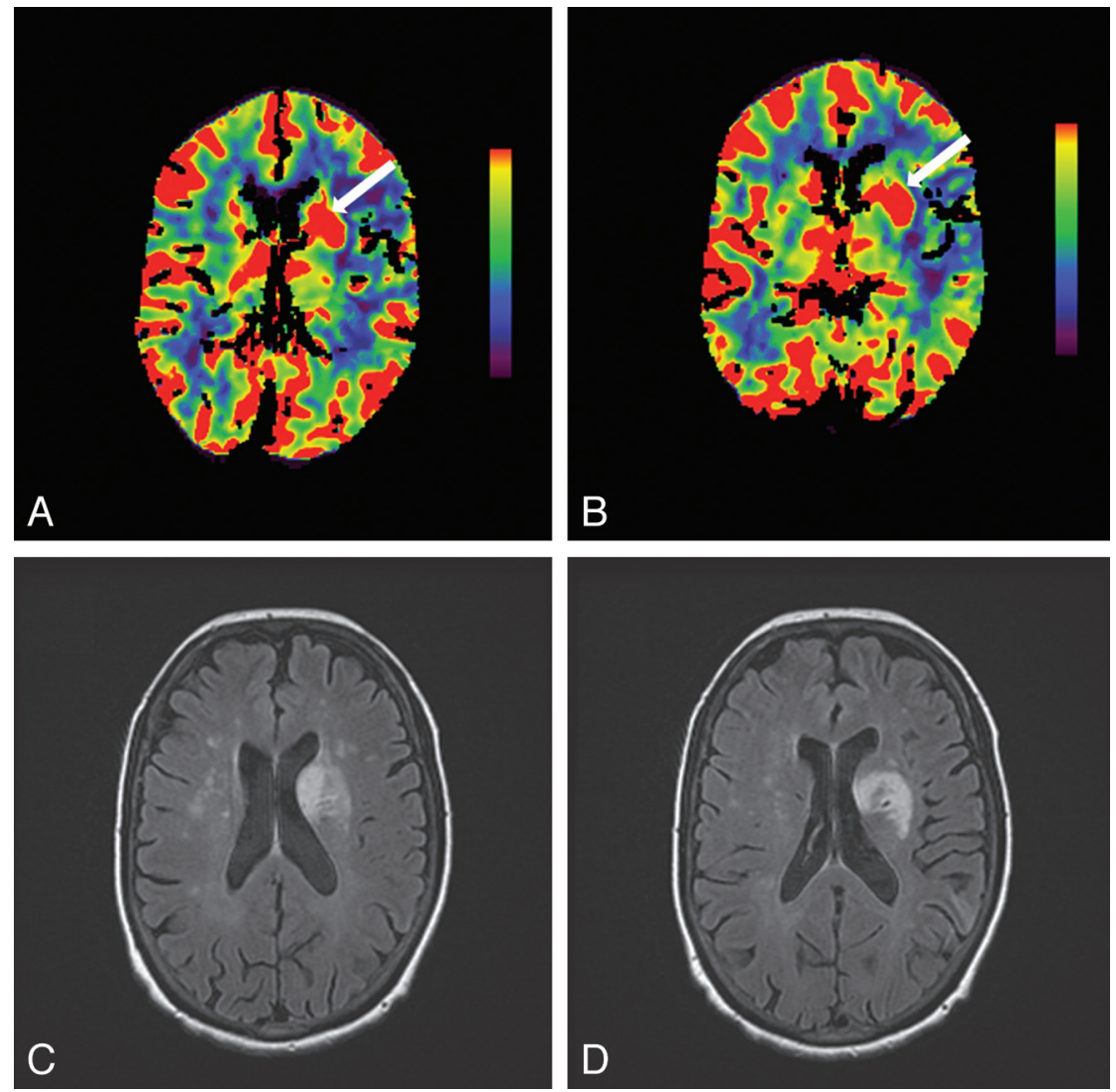

FIG 2. Hyperperfusion of the left basal ganglia is shown by increased cerebral blood flow (arrow, $A$ ) and increased cerebral blood volume (arrow, B) on CT perfusion. Follow-up FLAIR MR imaging 3.5 days later shows regions of T2 hyperintensity indicating infarction of this same region $(C$ and $D)$. involving superficial veins. In the latter case, areas of extravasation were also noted in addition to attenuated capillary blush. Endovascular recanalization was attempted in 5 cases and achieved partial $(n=1)$ or near-complete reperfusion $(n=3)$ in 4 of them.

Follow-up brain imaging was noncontrast head CT in 4 patients and MR imaging in 2. In all patients, the region of basal ganglia hyperperfusion proved to be infarcted on follow-up imaging (Figs 1-3). Two patients also had intracranial hemorrhage following recanalization (PH 2 in one case and $\mathrm{PH} 1$ in another). In both cases, the hemorrhage occurred in the basal ganglia, which had signs of hyperperfusion on the preintervention CTP. In the former case, early venous opacification into superficial veins, attenuated capillary blush, and areas of extravasation were noted, while the latter case had no angiographic abnormality suggestive of hyperperfusion or hemorrhage.

\section{DISCUSSION}

We present a series of patients with acute stroke who had evidence of basal ganglia hyperperfusion on CTP, which ultimately proved to be part of the infarction and/or a nidus for hemorrhagic transformation on follow-up imaging. All patients had received intravenous thrombolysis before CTP; CTA imaging at the time of CTP acquisition showed patency of the proximal M1 segment and Al segment, perhaps suggesting that partial recanalization, either spontaneous or in response to intravenous rtPA, may have resulted in luxury perfusion. Angiographic evidence of hyperperfusion, with early venous opacification, was present in a minority of cases.

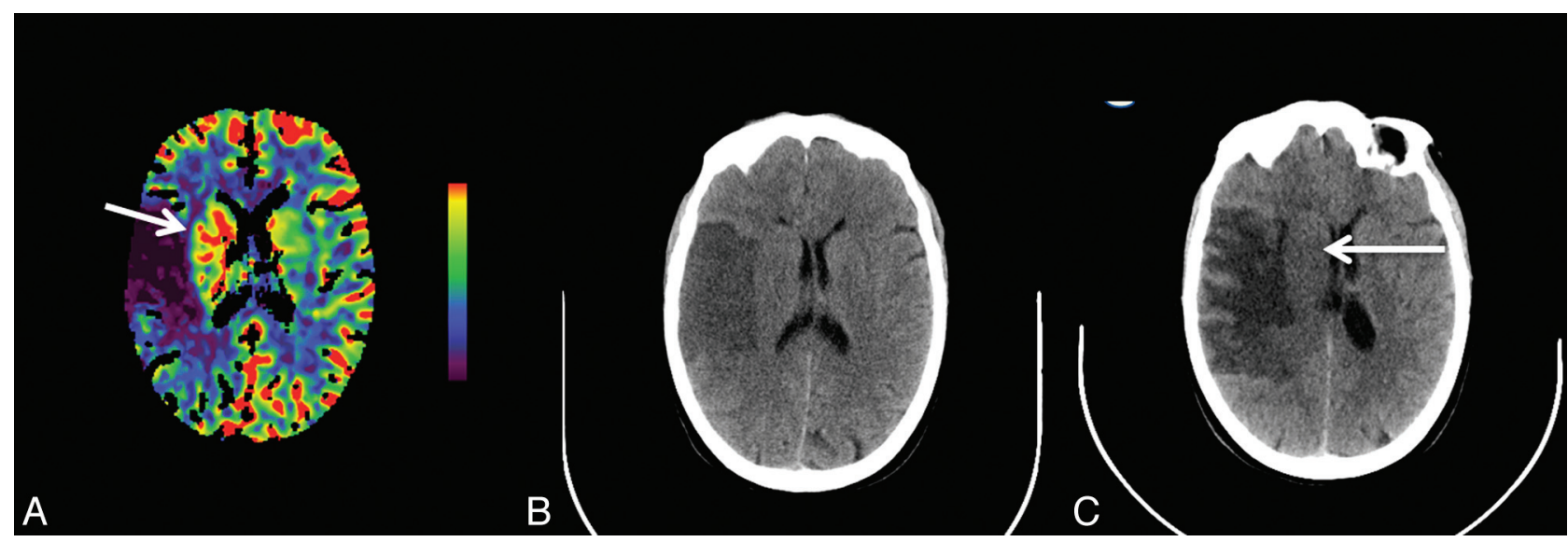

FIG 3. Increased cerebral blood flow in the right basal ganglia (arrow, $A$ ) in addition to a large region of reduced CBF in the right middle cerebral artery territory is shown on the CT perfusion scan at presentation. Noncontrast head CT 1 day later demonstrates right MCA infarction but apparent sparing of the right basal ganglia (B). Follow-up CT at day 6 shows hypoattenuation involving right basal ganglia (arrow, $C$ ) in addition to evolution of a large right MCA infarction with mass effect. 
Hemorrhagic transformation occurred in 2 patients, only one of whom had early venous opacification on angiography.

Our observation regarding hyperperfusion on CTP has potential clinical implications. First, practitioners should inspect CTP imaging for areas of hyperperfusion within the territory of the target artery, recognizing that these areas may be nonviable and may be at risk for hemorrhage. Failure to do this may cause underestimation of the ultimate size of the infarction and the risk of complications from mass effect when swelling develops. Furthermore, hyperperfusion in the basal ganglia may need to be considered part of the unsalvageable core when selecting appropriate candidates for endovascular therapy by using CTP, though our findings need to be confirmed in larger populations before this can be concluded with certainty. Advanced imaging with CTP is not necessary before the administration of IV thrombolysis for acute stroke, and we do not think that these findings should impact the decision to administer IV thrombolysis.

It is not exactly clear what the regions of hyperperfusion in the basal ganglia represent. This could be a result of partial recanalization of the M1 segment of the middle cerebral artery with a return of blood flow to blood vessels that had been maximally dilated because of ischemia. The basal ganglia receive blood from the lenticulostriate arteries, which usually arise from the M1 segment. On occasion, there is a truncated M1 segment due to normal anatomic variability, and the lateral lenticulostriate arteries may arise from the superior division of the M2 segment. The deep-seated basal ganglia may receive less collateral blood flow, have longer duration of ischemia compared with cortical regions, and thus may be more susceptible to reperfusion injury. Reperfusion after stroke has been associated with disruption of the bloodbrain barrier as evidenced by postgadolinium enhancement of CSF on MR imaging. ${ }^{13}$ This may be an indirect marker of reperfusion injury, which involves inflammation, increased cytokine release, excessive production of oxygen-free radicals, and formation of edema. Reperfusion injury is one reason why angiographic recanalization is not always associated with good clinical outcomes.

A "luxury perfusion syndrome" characterized by overabundant CBF relative to neuronal and glial metabolic demand is wellknown. ${ }^{8,9}$ Much of our knowledge of postischemic reperfusion is derived from experimental models, ${ }^{14}$ but the finding that postischemic hyperperfusion may include brain regions that become infarcted has also been demonstrated in small studies of human patients with stroke. ${ }^{6}$ In 1 study of patients with stroke who underwent large-vessel recanalization with intra-arterial fibrinolysis, hyperperfusion was demonstrated in $5 / 12$ by perfusion MR imaging several hours after recanalization. ${ }^{6}$ Hyperperfusion developed mainly in regions that subsequently were shown to be infarcted, and patients with hyperperfusion had larger final infarct volumes.

Others have produced discrepant results. A study by using PET within 5-18 hours of ischemic stroke showed that regions of hyperperfusion did not ultimately become infarcted on delayed follow-up imaging and suggested that hyperperfusion may not only be a harmless phenomenon but could even potentially be beneficial. ${ }^{15}$ Hyperperfusion in these cases predominantly affected patchy cortical regions remote from the deeper areas of ultimate infarction, with the limitation that all follow-up imaging was done with CT rather than MR imaging. These discrepant results across studies may be at least partially explained by differences in the time from arterial occlusion to reperfusion, the technique used to evaluate perfusion, and the type of follow-up brain imaging.

This study has several limitations. The small sample size and retrospective nature preclude definitive conclusions. This is a single-center study from a tertiary referral center, which may limit the generalizability of our findings. Our time to CTP was sometimes prolonged because most of our patients were treated at local emergency departments and then transferred to our center. The chances of finding basal ganglia hyperperfusion may be different if CTP is performed after a short interval from symptom onset. Finally, nearly all of our patients underwent endovascular recanalization, which may have affected the outcomes.

\section{CONCLUSIONS}

In acute stroke evaluations, basal ganglia hyperperfusion on CTP is an infrequent finding but important to recognize because it does not always predict a benign course.

These areas of hyperperfusion may represent irreversible ischemic damage and become the nidus for reperfusion hemorrhage, despite timely and successful recanalization therapy. These observations should be considered hypothesis-generating and require validation in larger prospective studies.

Disclosures: David F. Kallmes—UNRELATED: Consultancy: GE Healthcare,* ev3,* Comments: consulting on clinical trial planning and implementation, cost effectiveness research, Grants/Grants Pending: ev3, ${ }^{*}$ MicroVention, ${ }^{*}$ Micrus, ${ }^{*}$ Codman, ${ }^{*} \mathrm{Se}-$ quent, ${ }^{\star}$ Medina Medical, ${ }^{\star}$ NeuroSigma, ${ }^{\star}$ Surmodics, ${ }^{\star}$ Comments: funds for clinical trials and preclinical research, Royalties: University of Virginia Patent Foundation, Comments: Spine fusion. Alejandro A. Rabinstein-UNRELATED: Grants/Grants Pending: DJO Global, ${ }^{*}$ Comments: for investigator-initiated project on upper limb digital volume tomography, Royalties: Elsevier, Oxford, UptoDate, Comments: for authored books and chapters. *Money paid to the institution.

\section{REFERENCES}

1. Biesbroek JM, Niesten JM, Dankbaar JW, et al. Diagnostic accuracy of CT perfusion imaging for detecting acute ischemic stroke: asystematic review and meta-analysis. Cerebrovasc Dis 2013;35:493-501

2. Lin K, Do KG, Ong P, et al. Perfusion CT improves diagnostic accuracy for hyperacute ischemic stroke in the 3-hour window: study of 100 patients with diffusion MRI confirmation. Cerebrovasc Dis 2009;28:72-79

3. Campbell BC, Christensen S, Levi CR, et al. Cerebral blood flow is the optimal CT perfusion parameter for assessing infarct core. Stroke 2011;42:3435-40

4. Eastwood JD, Lev MH, Wintermark M, et al. Correlation of early dynamic CT perfusion imaging with whole-brain MR diffusion and perfusion imaging in acute hemispheric stroke. AJNR Am J Neuroradiol 2003;24:1869-75

5. Schramm P, Schellinger PD, Klotz E, et al. Comparison of perfusion computed tomography and computed tomography angiography source images with perfusion-weighted imaging and diffusionweighted imaging in patients with acute stroke of less than 6 hours' duration. Stroke 2004;35:1652-58

6. Kidwell CS, Saver JL, Mattiello J, et al. Diffusion-perfusion MRI characterization of post-recanalization hyperperfusion in humans. Neurology 2001;57:2015-21

7. Marchal G, Young AR, Baron JC. Early postischemic hyperperfusion: pathophysiologic insights from positron emission tomography. J Cereb Blood Flow Metab 1999;19:467-82 
8. Lassen NA. The luxury-perfusion syndrome and its possible relation to acute metabolic acidosis localised within the brain. Lancet 1966;2:1113-15

9. Ohta H. Postoperative luxury perfusion syndrome in patients with severe subarachnoid hemorrhage treated by early aneurysmal clipping. Neurol Med Chir (Tokyo) 1990;30:16-23

10. Nguyen TB, Lum C, Eastwood JD, et al. Hyperperfusion on perfusion computed tomography following revascularization for acute stroke. Acta Radiol 2005;46:610-15

11. Hacke W, Kaste M, Fieschi C, et al. Randomised double-blind placebo-controlled trial of thrombolytic therapy with intravenous alteplase in acute ischaemic stroke (ECASS II): Second EuropeanAustralasian Acute Stroke Study Investigators. Lancet 1998;352: 1245-51
12. Higashida RT, Furlan AJ, Roberts $H$, et al. Trial design and reporting standards for intra-arterial cerebral thrombolysis for acute ischemic stroke. Stroke 2003;34:e109-37

13. Warach S, Latour LL. Evidence of reperfusion injury, exacerbated by thrombolytic therapy, in human focal brain ischemia using a novel imaging marker of early blood-brain barrier disruption. Stroke 2004;35:2659-61

14. Heiss WD, Graf R, Lottgen J, et al. Repeat positron emission tomographic studies in transient middle cerebral artery occlusion in cats: residual perfusion and efficacy of postischemic reperfusion. J Cereb Blood Flow Metab 1997;17:388-400

15. Marchal G, Furlan M, Beaudouin V, et al. Early spontaneous hyperperfusion after stroke: a marker of favourable tissue outcome? Brain 1996;119:409-19 